\title{
Le Parquet Comme Acteur de la Justice en Republique Democratique du Congo
}

\author{
Symphorien Kapinga K. Nkashama ${ }^{1}$
}

\section{Résumé}

L'étude s'intéresse au Parquet comme l'un des acteurs de la justice en République Démocratique du Congo. L'auteur considère que cette institution est omniprésente dans l'espace judicaire congolais d'autant plus qu'on trouve le Parquet rattaché à chaque juridiction. Il joue à même temps le rôle de juge d'instruction et d'organe de poursuite dans le contentieux pénal, et donne des avis motivés sur toute question de droit soumise au juge dans les autres matières. Mais, le statut flottant du Parquet entre institution du pouvoir judiciaire et instrument du Gouvernement, sous l'autorité du Ministre de la justice, rend les magistrats du Parquet très vulnérables aux influences politiques.

\begin{abstract}
The study focuses on the prosecution as one of the actors of justice in the Democratic Republic of Congo. The author considers that this institution is omnipresent in the Congolese judicial area, especially since there is the prosecutor's office attached to each jurisdiction. At the same time, he plays the role of investigating judge and prosecutor in criminal litigation, and gives reasoned opinions on any question of law submitted to the judge in other matters. However, the floating status of the Procuratorate between the institution of the judiciary and the Government's instrument, under the authority of the Minister of Justice, makes public prosecutors highly vulnerable to political influence.
\end{abstract}

\section{Introduction}

Le Parquet est l'une des institutions omniprésentes dans l'administration de la justice en République Démocratique du Congo (RDC). Mais son statut juridique, son organisation et la mission sont assez complexes et difficiles à cerner dans la foulée des acteurs de la justice $^{2}$. Si historiquement le concept de Parquet désignait en France l'estrade qu'occupait le

1 Symphorien Kapinga K. Nkashama est détenteur du Diplôme d'Etudes Supérieures en Droit (Université de Kinshasa, Chef de travaux (Université de Mbujimayi) et Chercheur au Centre d'Etudes et des Recherches sur l'Etat de Droit en Afrique (Creeda).

2 Parmi les acteurs de la justice, on trouve au delà des magistrats du Parquet, des juges, des officiers de police judicaire, des greffiers et des huissiers de justice, des avocats et défenseurs judicaires... Lire à ces propos, Antoine Rubbens, Droit judicaire congolais, Tome I, Le pouvoir, l'organisation et la compétence judicaires, Kinshasa/Bruxelles, 1970, pp.159-226. 
représentant du Roi, lorsqu'il prenait parole devant le Juge, le Parquet renvoie aujourd'hui à un Office et se personnifie davantage aux membres du corps des magistrats, appelés tantôt « organes de la loi », tantôt «Officiers du Ministère public » ou «Procureurs de la République» dans le jargon judicaire congolais ${ }^{3}$.

D'après la Constitution du 18 février 2006 qui l'avait institué près chaque juridiction de l'ordre judicaire, de l'ordre administratif et même près la Cour constitutionnelle ${ }^{4}$, le Parquet faisait partie du pouvoir judiciaire. C'est ce qu'on peut lire à l'article 149 dans sa version initiale qui était rédigée ainsi:

Le pouvoir judiciaire est indépendant du pouvoir législatif et du pouvoir exécutif. Il est dévolu aux cours et tribunaux qui sont : la Cour constitutionnelle, la Cour de cassation, le Conseil d'Etat, la Haute Cour militaire ainsi que les Cours et Tribunaux civils et militaires ainsi que les parquets rattachés à ces juridictions ${ }^{5}$.

Cependant, depuis la révision constitutionnelle du 20 janvier 20116, œuvre de deux Chambres du Parlement réunies en congrès, le Parquet a été élagué de la liste des institutions auxquelles était dévolu le pouvoir judiciaire. Le constituant dérivé explique l'amendement de l'article 149 en ces termes:

L'amendement introduit à cet article consiste en la suppression du Parquet dans l'énumération des titulaires du pouvoir judiciaire. Celui-ci est dévolu aux seuls cours et tribunaux. Cet amendement remet ainsi en harmonie l'article 149 avec les articles 150 et 151 qui proclament l'indépendance du seul magistrat du siège dans sa mission de dire le droit ainsi que son inamovibilité 7 .

Certes, bien que supprimé par le Constituant dérivé en 2011, le Parquet a été institué de nouveau par le législateur près chaque juridiction, qu'elle soit de l'ordre judicaire, administratif et même près la Cour constitutionnelle, faisant du coup de cette institution, un acteur indispensable dans l'administration de la justice.

Sans vouloir se lancer dans le débat sur la conformité de cette loi de révision constitutionnelle aux conditions tant de forme que de fond prévues par la Constitution, notamment

3 Lire dans ce sens, Emmanuel-Janvier Luzolo Bambi Lessa, Traité de droit judiciaire. La justice congolaise et ses institutions, Kinshasa, 2018, p.105; Nkongolo Tshilengu, Droit judicaire congolais. Le rôle des cours et tribunaux dans la restauration d'un droit violé ou contesté, Kinshasa, 2003, p.95; Voir aussi Rubbens, note 2, p.185.

4 Article 149 al.1 et 2 de la Constitution du 18 février 2006, J.O.R.D.C., 47ème année, Numéro spécial du 18 février 2006.

5 Article 149 al. 1 et 2 de la Constitution du 18 février 2006 dans sa version initiale, note 4 .

6 Loi n ${ }^{\circ} 11 / 002$ du 20 janvier 2011 portant révision de certains articles de la Constitution de la République Démocratique du Congo, J.O.RDC, $52^{\text {ème }}$ année, Numéro spécial du 05 février 2011.

7 Exposé des motifs de la loi n $11 / 002$, note 6 . 
au regard de son article $220^{8}$ ni sur le statut juridique actuel du Parquet par rapport pouvoir judiciaire ou du pouvoir exécutif ${ }^{9}$, l'on peut pertinemment s'interroger sur le rôle de cette institution près chaque juridiction et dans l'administration de la justice? Comment est-il structuré? Comment est-il organisé? Quelles sont ses attributions? Voilà autant des questions que soulève le Parquet comme acteur de la justice et qui font l'objet de cette étude.

Nous essayons dans les lignes qui suivent d'étudier la composition, l'organisation et les attributions du Parquet près les juridictions de l'ordre judiciaire $(A)$, du Parquet près les juridictions de l'ordre administratif (B) et enfin, du Parquet près la Constitutionnelle (C) afin de cerner le rôle que cette institution est appelée à jouer dans l'administration de la justice.

\section{A. LE PARQUET PRES LES JURIDICTIONS DE L'ORDRE JUDICIAIRE}

Les juridictions de l'Ordre judiciaire sont : les tribunaux de paix, les tribunaux militaires de police, les tribunaux de grande instance, les tribunaux de commerce, les tribunaux du travail, les tribunaux militaires de garnison, les Cours militaires, les Cours militaires opérationnelles, les Cours d'appel, la Haute Cour militaire et la Cour de cassation ${ }^{10}$. Il convient de distinguer sur cette liste les juridictions de l'ordre judiciaire de droit commun des juridictions spécialisées ${ }^{11}$ qui sont créées par les lois spéciales, à savoir : les tribunaux militaires $^{12}$, les tribunaux de commerce ${ }^{13}$, les tribunaux de travail ${ }^{14}$ auxquels il faudra ajouter les tribunaux pour enfants ${ }^{15}$.

8 Lire à ce sujet, Auguste Mampuya Kanunk'a Tshiabo, « Au feu! L'article 220 est violé! », Le Phare du 13 janvier 2011 (Quotidien paraissant à Kinshasa).

9 Sur les controverses doctrinales, lire Luzolo Bambi Lessa, note 3, p.106. Marcel Wetsh'okonda Koso, "Nature juridique et garanties d'indépendance des officiers du ministère public sous l'empire de la Constitution congolaise du 18 février 2006 telle que révisée par la loi de révision constitutionnelle du 20 janvier 2011 ", Librairie Africaine d'Etudes Juridiques, 2017, p.533; Marcel Wetsh'okonda Koso, La protection des droits de l'homme par le juge constitutionnel congolais. Analyse critique et jurisprudence (2003-2013), Paris, 2016, pp.127-128; Bayona-ba-Meya, « L'indépendance de la magistrature en République du Zaïre », Annales de la Faculté de droit, Vol. I, 1972, p.32.

10 Article 6 de la Loi organique $\mathrm{n}^{\circ}$ 13/011-B du 11 avril 2013 portant organisation, fonctionnement et compétences des juridictions de l'ordre judicaire, J.O.R.D.C., $59^{\text {ème }}$ année, Numéro spécial 7, juin 2018, pp.249-282.

11 Elles sont créées conformément à l'article 149 alinéa 5 de la Constitution, note 4 .

12 Loi n $023-2002$ du 18 novembre 2002 portant code justice militaire, J.O.R.D.C, $43^{\text {ème }}$ année, du 20 mars 2003, Numéro spécial, p. 1.

13 Loi n ${ }^{\circ} 002 / 2001$ du 03 juillet 2001 portant création, organisation et fonctionnement des Tribunaux de commerce, $42^{\text {ème }}$ année, J.O.R.D.C. n ${ }^{\circ} 14$ du 15 juillet 2001, p.4.

14 Loi n ${ }^{\circ} 016-2002$ du 16 octobre 2002 portant création, organisation et fonctionnement des Tribunaux du travail, J.O.R.D.C.,.

15 Loi nº9/001 du 10 janvier 2009 portant protection de l'enfant, J.O.R.D.C., $50^{\text {ème }}$ année, Numéro spécial 12 janvier 2009, pp.13-48. 


\section{Composition et organisation du Parquet près les juridictions de l'ordre judiciaire}

Près chaque juridiction de l'ordre judiciaire, il est institué un Parquet ${ }^{16}$. Les structures des parquets comme celles des juridictions auxquelles ils sont attachés, sont prévues par l'Arrêté d'organisation judiciaire n²99/79 du 20 août 1979 portant règlement intérieur des cours, Tribunaux et Parquets. Nous essayerons de présenter la composition du Parquet près les juridictions judiciaires (1) et la hiérarchie au sein de cette institution (2).

\section{Composition du Parquet}

La composition du Parquet près les juridictions de l'ordre judicaire diffère suivant chaque juridiction. Au niveau du Conseil d'Etat, le Parquet près cette juridiction est constitué du Procureur général, assisté d'un ou de plusieurs Premiers avocats généraux et d'un ou plusieurs Avocats généraux ${ }^{17}$.Ceux-ci exercent les mêmes fonctions et ont donc les mêmes prérogatives que le Procureur général sous la surveillance et la direction de ce dernier.

Au niveau des Cours d'appel, le Parquet près cette juridiction est dirigé par un Procureur général assisté d'un ou plusieurs Avocats généraux et d'un ou plusieurs Substituts du Procureur général qui exercent les prérogatives de ce dernier sous sa surveillance et sa direction $^{18}$.

Tandis que les Parquets près les Tribunaux de grande instance sont sous la responsabilité du Procureur de la République. Celui-ci est assisté par est assisté d'un ou de plusieurs Premiers Substituts et d'un ou plusieurs Substituts du Procureur de la République ${ }^{19}$.

Au niveau des Tribunaux de paix, il n'y avait pas de Parquet près ces juridictions, les fonctions de ministère public étaient exercées par le Procureur de la République près le Tribunal de grande instance du ressort ${ }^{20}$. Mais, depuis la réforme de 2013, le législateur a désormais institué un Parquet près chaque tribunal de paix. Celui-ci est placé sous la direction d'un Premier substitut du Procureur de la République auquel sont adjoints un ou plusieurs substituts du Procureur de la République ${ }^{21}$.

Comme on peut le remarquer, il n'est pas institué de Parquet près le Tribunaux de commerce, les Tribunaux de travail ainsi que les tribunaux pour enfant qui sont des juridictions spécialisées de l'ordre judiciaire. Mais, le Procureur de la République près le Tribunal de grande instance dans le ressort duquel se trouvent le tribunal de commerce, le Tribunal du

16 Article 65 de la Loi organique ${ }^{\circ} 13 / 011-\mathrm{B}$, note 6 .

17 Article 65 de la Loi organique $n^{\circ} 13 / 011-\mathrm{B}$, note 6.

18 Article 65 de la Loi organique ${ }^{\circ} 13 / 011-\mathrm{B}$, note 6 .

19 Article 65 de la Loi organique $n^{\circ} 13 / 011-B$, note 6 .

20 Article 16 alinéa1 de l'Ordonnance-Loi portant n82-020 du 31 mars 1982 portant code de l'organisation et des compétences judicaires, $J O Z, 23^{\text {ème }}$ année, numéro spécial.

21 Article 65 de la Loi organique $n^{\circ} 13 / 011-\mathrm{B}$, note 6 . 
travail ou le Tribunal pour enfant exerce les fonctions du Ministère public près ces juridictions $^{22}$.

Il convient aussi de noter que les magistrats du Parquet comme ceux du siège sont nommés et relevés de leurs fonctions, le cas échéant, révoqués par le Président de la République sur proposition du Conseil Supérieur de la Magistrature ${ }^{23}$. Ils sont recrutés sur concours conformément à la loi portant statut des magistrats.

\section{Hiérarchie du Parquet}

Bien que les structures du Parquet soient aujourd'hui modelées suivant les juridictions auxquels il est appelé à exercer les fonctions, le principe hiérarchique et l'unicité du Ministère public $^{24}$ domine en réalité ce morcellement formel. Le Parquet ou Ministère public remplit les devoirs de son office auprès des juridictions établies dans son ressort ${ }^{25}$.

Ainsi, le Procureur général près la Cour de cassation exerce les fonctions du Ministère Public près cette juridiction, en ce compris l'action publique. Il peut cependant, sur injonction du Ministre de la justice : initier ou continuer toute instruction préparatoire portant sur des faits infractionnels qui ne ressortent pas de la compétence de la Cour de cassation, requérir et soutenir l'action publique devant tous les Cours et Tribunaux à tous les niveaux. Il peut également, sur injonction du Ministre de la justice, ou d'office et pour l'exécution des mêmes devoirs faire injonction aux Procureurs généraux près la Cour d'appel ${ }^{26}$.

Le Procureur général près la Cour de Cassation a un droit de surveillance et d'inspection sur les Parquets généraux près les Cours d'appel. Il peut, à ce titre, demander et recevoir en communication tout dossier judiciaire en instruction à l'office du Procureur général près la Cour d'appel ou à celui du Procureur de la République. Il ne peut cependant, à peine de nullité de la procédure, poser des actes d'instruction ou de poursuite dans le dossier reçu en communication que sur injonction du Ministre de la Justice ${ }^{27}$.

Il convient néanmoins de souligner que l'exercice de l'action publique dans toute sa plénitude et devant toutes les juridictions du ressort de la Cour d'Appel appartient au Procureur général près cette Cour. Le Procureur Général prend la parole aux audiences solennelles de la Cour d'appel. Il peut aussi le faire aux audiences des chambres s'il le juge nécessaire ${ }^{28}$.

22 Articles 13 de la Loi ${ }^{\circ} 016-2002$, note 13 , article 12 de la loi $n^{\circ} 002$, note 13 , article 92 de la Loi $n$ ${ }^{\circ} 09$, note 15 .

23 Article 82 de la Constitution, note 4.

24 Luzolo Bambi Lessa, note 3, p.171.

25 Article 71 de la Loi organique $\mathrm{n}^{\circ} 13 / 011-\mathrm{B}$, note 6 .

26 Article 72 de la Loi organique $n^{\circ} 13 / 011-B$, note 6.

27 Article 73 de la de la Loi organique $n^{\circ} 13 / 011-\mathrm{B}$, note 6.

28 Article 77 de la Loi organique $n^{\circ} 13 / 011-\mathrm{B}$, note 6. 
Mais dans les relations entre le Parquet et le pouvoir exécutif, le législateur stipule ceci:

Les officiers du Ministère Public sont placés sous l'autorité du Ministre ayant la justice dans ses attributions. Celui-ci dispose d'un pouvoir d'injonction sur le Parquet. Il l'exerce en saisissant le Procureur général près la Cour de cassation ou le Procureur général près la Cour d'appel selon le cas sans avoir à interférer dans la conduite de l'action publique ${ }^{29}$.

Est-ce pour autant que les magistrats du Parquet près les juridictions de l'ordre judicaire deviennent de simples agents de l'administration publique à la disposition du Ministre de la justice? Quel serait l'étendue de l'autorité du Ministre de la justice sur les magistrats du Parquet qui sont encore régis par le statut des magistrats et qui sont placés sous l'autorité disciplinaire du Conseil Supérieur de la Magistrature? La question fait débat dans la doctrine depuis l'exclusion du Parquet du pouvoir judiciaire suite à la révision de l'article 149 alinéa 2 de la Constitution.

Interprétant la disposition légale ci-dessus qu'il n'a pas daigné reprendre in extenso, Luzolo Bambi Lessa, conclut hâtivement en ces termes :

L'autorité implique la subordination de la part du subordonné vis-à-vis du chef hiérarchique. L'autorité dont est revêtu le ministre de la justice sur les magistrats du Parquet fait de lui le chef de tous ces magistrats ${ }^{30}$.

A notre avis, cette conclusion n'est pas du tout convaincante d'autant plus que l'autorité du Ministre de la justice sur les magistrats du Parquet est bien circonscrite et limitée. En effet, le législateur a pris les soins de préciser dans la même disposition légale in fine ce pouvoir d'injonction du Ministre comme suit:

Il l'exerce en saisissant le Procureur général près la Cour de cassation ou le Procureur général près la Cour d'appel selon le cas sans avoir à s'interférer dans la conduite de l'action publique ${ }^{31}$.

Commentant d'ailleurs le pouvoir d'injonction du Ministre de la justice sur le Parquet, Antoine Rubbens relevait déjà avec raison que, suivant le rapport du Conseil colonial rédigé par SOHIER (B.O., 58, p.20), ceci veut dire :

L'exercice de l'action publique est attribué directement au Procureur général; le Ministre ne peut l'entamer par un veto; mais le Ministre a le droit d'injonction, c'est-à-

29 Article 70 de la Loi organique $\mathrm{n}^{\circ} 13 / 011-\mathrm{B}$, note 6 .

30 Luzolu Bambi Lessa, note 3, p.106.

31 Article 70 in fine de la Loi organique ${ }^{\circ} 13$, note 6 . On trouve les mêmes principes à l'article $15 \mathrm{de}$ la Loi organique $n^{\circ} 06 / 20$ du 10 octobre 2006 portant statut des magistrats telle modifiée par la Loi organique $\mathrm{n}^{\circ} 15 / 014$ du 1er août 2015, J.O.R.D.C., 56ème année, n 17 , 1er septembre 2015. Lire dans ce sens, Marcel Wetsh'okonda Koso, note 9, p.547-548. 
dire qu'il peut prescrire au Procureur général de faire usage de ses pouvoirs légaux d'instruction et de poursuite ${ }^{32}$.

Et l'auteur de conclure en ces termes :

Le Ministre dispose donc d'un droit d'impulsion; il ne peut pas se substituer aux officiers du Ministère public pour agir en leurs lieu et place; il ne peut arrêter l'action publique mise en mouvement par le parquet ${ }^{33}$.

\section{Attributions du Parquet}

Les magistrats du Parquet près les juridictions de l'ordre judicaire exercent la fonction du Ministre public près ces juridictions de l'ordre judiciaire. Ils participent ainsi à l'administration de la justice quand bien même leur rôle diffère selon qu'on se trouve dans une affaire civile ou pénale ainsi que dans les matières extrajudiciaires.

\section{En matière répressive $e^{34}$}

En matière pénale, le Parquet ou le Ministère public joue à même temps le rôle de juge d'instruction et d'organe de poursuite. Comme juge d'instruction, le Parquet est chargé recherche les infractions aux actes législatifs et réglementaires qui sont commises sur le territoire de la République. A cet effet, le Parquet reçoit des plaintes et des dénonciations, accomplit tous les actes d'instruction en réunissant des preuves et saisit les cours et tribunaux. Il s'appuie sur les officiers de police judicaire éparpillés sur l'ensemble du territoire national qui sont ses yeux et ses oreilles ${ }^{35}$.

Pour accomplir sa mission, le Parquet dispose du pouvoir d'arrêter et de placer en détention provisoire les personnes suspectées d'avoir commis des faits punissables de 6 mois de servitude pénale au moins, et sur lesquelles pèsent les indices sérieux de culpabilité ${ }^{36}$.

Devant le Tribunal, le Ministère public est partie au procès pénal au même titre que le prévenu. Il pose des questions, présente ses réquisitoires pour soutenir l'action pénale devant les cours et tribunaux ${ }^{37}$.

32 Rubbens, note 2, p.181.

33 Rubbens, note 2, p.181.

34 Pour plus des détails, voir Emmanuel -Janvier Luzolu Bambi Lessa et Nicolas Abel Bayona Ba Meya, Manuel de Procédure pénale, Kinshasa, 2011, pp.208 et suiv.

35 Article 1 Décret du 06 août 1959 portant code de procédure pénale, B.O. 1959, p.194.

36 Article 27 du Décret du 06 août 1959, note 32.

37 Article 67 de la Loi-organique $n^{\circ} 13, / 011-B$ note 6. 


\section{En matière de droit privé}

En matière de droit privé, le Ministère public intervient soit par voie d'avis, soit par voie d'action. Il donne obligatoirement son avis dans les cas prévus par la loi ${ }^{38}$. Il convient de relever que l'avis du Ministère Public est donné par écrit dans les trente jours après que la cause lui ait été communiquée, à moins qu'en raison des circonstances de l'affaire, il puisse être émis verbalement sur les bancs. Dans ce cas, l'avis est acté à la feuille d'audience. Si l'avis n'est pas donné dans ce délai, le chef de la juridiction fait obligation au chef d'office de ramener le dossier en l'état et la cause est prise en délibéré. La décision rendue mentionne que l'avis du Ministère Public n'a pas été donné dans le délai ${ }^{39}$.

Il peut agir par voie d'action principale dans l'intérêt de toute personne physique lésée qui serait inapte à ester en justice, à assurer sa défense et à y pourvoir ${ }^{40}$. Il peut par voie de requête écrite, demander au Président de la juridiction, la désignation d'un conseil ou d'un défenseur chargé d'assister les personnes visées à l'alinéa précédent. Il agit d'office comme partie principale ou intervenante dans les cas spécifiés par la loi et chaque fois que l'intérêt public exige son concours ${ }^{41}$.

\section{Autres attributions}

Le Ministère public surveille l'exécution des actes législatifs, des actes réglementaires et des décisions de justice ${ }^{42}$. Il poursuit d'office cette exécution dans les dispositions qui intéressent l'ordre public ${ }^{43}$. Il a la surveillance de tous les officiers de police judiciaire, des officiers publics et des officiers ministériels, sauf des agents du greffe et de l'office des huissiers $^{44}$.

Le Ministère public veille au maintien de l'ordre dans les cours et tribunaux sans préjudice des pouvoirs du juge qui a la police de l'audience ${ }^{45}$. Il assiste à toutes audiences de la Cour de cassation, des cours d'appel, des tribunaux de grande instance, des tribunaux de commerce, des tribunaux de travail et des tribunaux de paix. Il ne prend pas part au délibéré 46 .

38 Article 69 alinéa 1 de la Loi organique $\mathrm{n}^{\circ} 13 / 011-\mathrm{B}$, note 6.

39 Article 69 alinéa 2 de la Loi organique, n¹3/011-B, note 6.

40 Article 68 alinéas 1 à 3 de la Loi-organique n $13 / 011-\mathrm{B}$, note 6 .

41 Article 68 alinéas 4 et 5 de la Loi organique n¹3/011-B, note 6 .

42 Article 66 alinéa 1 de la Loi-organique ${ }^{\circ} 13 / 011-\mathrm{B}$, note 6.

43 Article 66 alinéa 2 de la Loi-organique ${ }^{\circ} 13 / 011-\mathrm{B}$, note 6.

44 Article 66 alinéa 3 de la Loi-organique ${ }^{\circ} 13 / 011-\mathrm{B}$, note 6.

45 Article 66 alinéa 4 de la Loi-organique ${ }^{\circ} 13 / 011-\mathrm{B}$, note 6.

46 Article 66 alinéa 5 de la Loi-organique n¹3/011-B, note 6. 


\section{B. PARQUET PRES LES JURIDICTIONS DE L'ORDRE ADMNISTRATIF}

L'ordre des juridictions administratives comprend les juridictions de droit commun et les juridictions spécialisées ${ }^{47}$. Les juridictions de droit commun sont : le Conseil d'Etat, les Cours administratives d'appel et les tribunaux administratifs ${ }^{48}$.

Tandis que les juridictions spécialisées de l'ordre administratif sont celles créées et organisées en vertu de l'article 149 alinéa 6 de la Constitution ${ }^{49}$. Comme près les juridictions de l'ordre judiciaire, le législateur a institué un Parquet près les juridictions de l'ordre administratif du moins pour les juridictions administratives de droit commun ${ }^{50}$.

\section{COMPOSITION ET ORGANISATION DU PARQUET}

\section{Composition}

Au niveau du Conseil, il est institué un Parquet Général près cette juridiction. Celui-ci est dirigé par un Procureur général près le Conseil d'Etat, assisté d'un ou de plusieurs Premiers Avocats généraux et d'un ou plusieurs Avocats généraux ${ }^{51}$. Près chaque Cour administrative d'appel, il est rattaché un Parquet général placé sous la direction d'un Procureur général près la Cour administrative d'appel, assisté d'un ou de plusieurs Avocats généraux et d'un ou plusieurs Substituts du Procureur général ${ }^{52}$.

Tandis qu'au niveau de chaque tribunal administratif, il est rattaché un Parquet composé d'un Procureur de la République, d'un ou de plusieurs Premiers Substituts du Procureur et d'un ou de plusieurs substituts du Procureur de la République ${ }^{53}$. Tous les membres du Parquet sont soumis au statut des magistrats.

\section{Organisation}

Comme pour le Parquet près les juridictions de l'ordre judicaire, le Ministère public remplit les devoirs de son office auprès de juridictions établies dans son ressort ${ }^{54}$. Ainsi, le Procureur général près le Conseil d'Etat exerce les fonctions du Ministère public près cette juri-

47 Article 2 alinéa 1 de la Loi organique ${ }^{\circ} 16 / 027$ du 15 octobre 2016 portant organisation, compétence et fonctionnement des juridictions de l'ordre administratif, J.O.R.D.C., $59^{\text {ème }}$ année, Numéro spécial du 7 juin 2018, pp.289-282.

48 Article 2 alinéa 2 de la Loi organique ${ }^{\circ} 16 / 027$, note 46.

49 Article 2 alinéa 3 de la Loi organique $n^{\circ} 16 / 027$, note 46 . Il s'agit de la Cour des comptes et des juridictions disciplinaires des administrations publiques ou des ordres professionnels.

50 Article 32 de la Loi organique $\mathrm{n}^{\circ} 16 / 027$, note 46 .

51 Article 36 alinéa 1 de la Loi organique $n^{\circ} 16 / 027$, note 46 .

52 Article 37 alinéa 1 de la Loi organique $n^{\circ} 16 / 027$, note 46 .

53 Article 39 alinéa 1 de la Loi organique $n^{\circ} 16 / 027$, note 46.

54 Article 35 de la Loi organique ${ }^{\circ} 16 / 027$, note 46 . 
diction. Les Premiers Avocats généraux et les Avocats généraux exercent les fonctions du ministère public sous sa surveillance et sa direction ${ }^{55}$.

Le Procureur général près le Conseil d'Etat dispose du droit de surveillance et d'inspection sur les parquets généraux près les cours administratives d'appel et sur les Parquets près les Tribunaux administratifs ${ }^{56}$. Le Procureur près la Cour administrative d'appel exerce les fonctions du Ministère public près cette juridiction. Les Avocats généraux et les substituts du Procureur général exercent leurs fonctions sous sa surveillance et sa direction ${ }^{57}$. Il dispose du droit de surveillance et d'inspection sur les Parquets près les tribunaux administratifs de son ressort ${ }^{58}$.

Le Procureur de la République près le Tribunal administratif exerce, sous la surveillance et la direction du Procureur général près la Cour administrative d'appel, les fonctions du ministère public. Les Premiers substituts et les Substituts du Procureur de la République exercent leurs fonctions sous sa surveillance et sa direction ${ }^{59}$.

\section{ATTRIBUTIONS}

Devant les juridictions administratives, le Ministère public intervient par voie d'avis. Il agit aussi par voie d'action dans les cas de renvoi pour cause de sureté publique, de révision ou pourvoi dans l'intérêt de la loi. Il participe aux audiences des cours et tribunaux mais ne prend pas part au délibéré ${ }^{60}$.

Dans l'exercice da sa mission, l'Officier du Ministère public près les juridictions de l'ordre administratif expose publiquement et en toute indépendance son opinion sur les questions que les requêtes présentent à juger et les solutions qu'elles appellent ${ }^{61}$.

\section{PARQUET PRES LA COUR CONSTITUTIONNELLE}

Comme pour les juridictions de l'ordre judiciaire et celles de l'ordre administratif, le législateur a institué près la Cour constitutionnelle un Parquet Général. ${ }^{62}$. Nous essayons dans les lignes qui suivent de voir sa raison d'être (I) sa composition (I) ainsi ses attributions (III).

55 Article 36 alinéa 2 de la Loi organique $n^{\circ} 16 / 027$, note 46 .

56 Article 36 alinéa 3 de la Loi organique $n^{\circ} 16 / 027$, note 46 .

57 Article 37 alinéa 2 de la Loi organique $n^{\circ} 16 / 027$, note 46 .

58 Article 37 alinéa 4 de la Loi organique $n^{\circ} 16 / 027$, note 46 .

59 Article 39 alinéa 2 de la Loi organique $n^{\circ} 16 / 027$, note 46.

60 Article 33 de la Loi organique ${ }^{\circ} 16 / 027$, note 46 .

61 Article 34 de la Loi organique ${ }^{\circ} 16 / 027$, note 46 .

62 Article 12 alinéa 1 de la Loi organique n $13 / 026$ du 15 octobre 2013 portant organisation et fonctionnement de la Cour constitutionnelle, J.O.R.D.C., $54^{\text {ème }}$ année, Numéro spécial, 18 octobre 2016. 


\section{Raison d'être et composition du parquet près la Cour constitution}

1. Raison d'être du Parquet près la Cour constitutionnelle

Pourquoi avoir institué le Parquet près la Cour constitutionnelle? Cette question sur le bien fondé de cette institution vaut son pesant d'or d'autant plus que, on ne trouve pas généralement de parquet à coté des cours constitutionnelles ou Conseils constitutionnels de la plupart des Etats au monde. La loi organique ayant institué le parquet général près la Cour constitutionnelle ne donne pas clairement la raison d'être de cette institution; le législateur se contentant d'annoncer sa création dans l'exposé des motifs sans en fournir d'amples explications.

Cependant, l'on peut facilement déduire l'existence du Parquet près la Cour constitutionnelle des compétences pénales reconnues à celle-ci. En effet, contrairement à la plupart des juridictions constitutionnelles d'autres pays, la Cour constitutionnelle congolaise est :

le juge pénal du Président de la République et du Premier Ministre pour les infractions politiques de haute trahison, d'outrage au Parlement, d'atteinte à l'honneur ou à la probité ainsi que pour les délits d'initiés et pour les autres infractions de droit commun commises dans l'exercice ou à l'occasion de l'exercice de leurs fonctions ${ }^{63}$.

Comme on peut le deviner, c'est principalement à cause des compétences pénales reconnues à la Cour constitutionnelle que le Parquet, s'est imposé près cette juridiction, pour y exercer les fonctions du Ministère public, chargé de mettre en mouvement l'action publique contre le Président de la République ou le Premier Ministre ${ }^{64}$.

La présence du Parquet près la Cour constitutionnelle est aussi la suite logique de l'option levée par le constituant d'éclater les Cours et tribunaux en trois ordres juridictionnels auxquels est rattaché un parquet pour plus d'efficacité, de spécialisation et de célérité dans le traitement des dossiers ${ }^{65}$. Ainsi, logiquement, les magistrats du parquet rattaché à un ordre juridictionnel ne peuvent pas prester devant les juridictions d'un autre ordre ${ }^{66}$.

\section{Composition du Parquet près la Cour constitutionnelle}

Le Parquet Général près la Cour constitutionnelle est placé sous l'autorité du Procureur général ${ }^{67}$, assisté d'un ou de plusieurs Premiers Avocats Généraux et d'un ou plusieurs Avocats généraux. Ils sont nommés par le Président de la République sur proposition du

63 Article 164 de la Constitution, note 4.

64 Lire dans ce sens Jean-Louis Essambo Kangashe, La Constitution congolaise du 18 février 2006 à l'épreuve du constitutionnalisme. Contraintes pratiques et perspectives, 2010, Louvain-la-Neuve, p.255.

65 Exposé des motifs de la Constitution, note 4.

66 Lire dans ce sens Essambo Kangashe, note 63, p.255.

67 Article 12 alinéa 2 de la Loi organique $n^{\circ} 13 / 026$, note 61 . 
Conseil supérieur de la magistrature, pour un mandat de trois ans renouvelable une fois. Ils sont soumis au statut des membres de la Cour constitutionnelle ${ }^{68}$.

Les membres du Parquet général près la Cour constitutionnelle sont recrutés parmi les magistrats de l'ordre judiciaire ou administratif ayant au moins quinze ans d'expérience ${ }^{69}$. A notre avis, cette exigence est en contradiction avec l'option de spécialisation des juridictions levée par le Constituant. Comment recruter parmi les magistrats de l'ordre judiciaire ou administratif des spécialistes de la justice constitutionnelle?

Avant leur entrée en fonction, les membres du Parquet prêtent le serment ci-après devant le Président de la République : « Je jure de respecter la Constitution et les lois de la République Démocratique du Congo et de remplir loyalement et fidèlement, avec honneur et dignité, les fonctions qui me sont confiées ${ }^{70}$.

La prestation du serment des membres du Parquet est une formalité substantielle. Lorsqu'elle fait défaut, les actes posés par le membre du Parquet comme celui de la Cour qui n'a pas prêté serment sont nuls et de nullité absolue.

Pour réaliser sa mission, le Parquet Général près la Cour constitutionnelle est doté d'un Secrétariat général, dont les attributions, l'organisation et le fonctionnement sont déterminés par Décret du Premier Ministre ${ }^{71}$. Dans le cadre de la collaboration horizontale entre Parquets, Il peut recourir aussi aux services du Parquet près la Cour de cassation, moyennant une réquisition ${ }^{72}$.

\section{Attributions du Parquet près la Cour constitutionnelle}

Comme pour tout autre parquet près les juridictions de l'ordre judiciaire ou administratif, la présence des membres du Parquet près la Cour constitutionnelle, faisant office du Ministère public aux audiences de cette juridiction, est une condition sine qua non pour la composition régulière de du siège ${ }^{73}$. Mais le rôle du Parquet diffère selon qu'on se trouve dans le contentieux pénal ou dans les autres contentieux qui relèvent de la compétence de la Cour constitutionnelle.

a) Rôle du Parquet dans le contentieux pénal

En matière pénale, le Parquet près la Cour constitutionnelle joue le rôle d'organe d'instruction et de poursuites devant cette juridiction comme tous les autres parquets. Mais il ne re-

68 Article 13 de la Loi organique $n^{\circ} 13 / 026$, note 61.

69 Article 13 de la Loi organique $n^{\circ} 13 / 026$, note 61.

70 Article 16 de la Loi organique ${ }^{\circ} 13 / 026$, note 61 .

71 Article 17 de la Loi organique $n^{\circ} 13 / 026$, note 61 .

72 Article 18 de la Loi organique, $\mathrm{n}^{\circ} 13 / 026$, note 61 .

73 Article 14 alinéa 2 de la Loi organique $n^{\circ} 13 / 026$, note 61 . 
cherche et ne constate que les infractions relevant de la compétence de la Cour constitutionnelle pour lesquelles il soutient l'accusation et requiert les peines ${ }^{74}$.

Ainsi, le Procureur Général près la Cour constitutionnelle assure l'exercice de l'action publique dans les actes d'instruction et de poursuites contre le Président de la République, le Premier Ministre ainsi que les co-auteurs et leurs complices ${ }^{75}$. A cette fin, il reçoit les plaintes et les dénonciations et rassemble les preuves. Il entend toute personne susceptible de contribuer à la manifestation de la vérité 76 .

Si le Procureur Général près la Cour constitutionnelle estime devoir poursuivre le Président de la République ou le Premier Ministre, il sollicite du Parlement l'autorisation des poursuites ${ }^{77}$. Celle-ci est votée par le Parlement réuni en congrès à la majorité de deux tiers des membres du Parlement ${ }^{78}$. A la clôture de l'instruction préjuridictionnelle, le Procureur Général adresse le rapport au Président de l'Assemblée et du Sénat, éventuellement accompagné d'une demande d'autorisation de la mise en accusation du Président de la République ou du Premier Ministre ${ }^{79}$. L'autorisation de mise en accusation est votée par le Parlement dans les mêmes formes que l'autorisation de poursuites ${ }^{80}$.

Si le Congrès autorise la mise en accusation du Président de la République ou du Premier Ministre, le Procureur Général transmet le dossier, par une requête aux fins de fixation d'audience, le dossier à la Cour. Il fait citer les prévenus et, s'il ya lieu, les co-auteurs et/ou les complices ${ }^{81}$. Comme dans la procédure pénale ordinaire, le Parquet près la Cour constitutionnelle est partie au procès et y participe activement. Il soutient son action et prend des réquisitoires pour démontrer le bien fondé de son action et requiert les peines contre les prévenus.

Cependant, il convient de noter que, contrairement aux autres Parquets, le Procureur Général près la Cour n'a pas le pouvoir ni d'arrêter ni de placer en détention le Président de la République ou le Premier Ministre qui font l'objet des poursuites. Seule la Cour peut autoriser la mise en détention dont les modalités dans chaque cas. Celle-ci est remplacée par l'assignation à résidence surveillée ${ }^{82}$.

74 Article 14 alinéa 1 de la Loi organique $n^{\circ} 13 / 026$, note 61 .

75 Article 100 de la Loi organique $n^{\circ} 13 / 026$, note 61 .

76 Article 100 de la Loi organique, ${ }^{\circ} 13 / 026$, note 61. Pour d'autres commentaires, lire Guy-Prosper Djuma Bilali Lokema, «Parquet Général près la Cour constitutionnelle de la République Démocratique du Congo », Annuaire Congolais de Justice Constitutionnelle, Vol.1, 2016, p.98.

77 Article 100 de la Loi organique $n^{\circ} 13 / 026$, note 61 .

78 Article 166, alinéa 1 de la Constitution, note 4.

79 Article 103, alinéa 1 de la Loi organique n¹3/026, note 61.

80 Article 166, alinéa 1 de la Constitution, note 4.

81 Article 103 alinéa 2 de la Loi organique $n^{\circ} 13 / 026$, note 61.

82 Article 102 alinéa 2 de la Loi organique $n^{\circ} 13 / 026$, note 61 . 
b. Rôle du Parquet dans les autres contentieux

En dehors du contentieux pénal, le Parquet près la Cour constitutionnelle joue aussi le rôle du Ministère public dans toutes les autres affaires qui relèvent de la compétence de cette Cour. Il s'agit du recours en conformité à la Constitution, du contentieux d'inconstitutionnalité, de l'interprétation de la Constitution, des conflits de compétences ou d'attributions, du contentieux électoral et des autres matières non contentieuses soumises à la Cour constitutionnelle.

Dans toutes ces matières autres que le contentieux pénal qui relèvent de la Cour constitutionnelle, le Procureur Général émet des avis motivés. Il assiste à toutes les audiences et peut y présenter des observations. Mais, il ne prend pas part au délibéré ${ }^{83}$.

Comme on peut bien le remarquer, dans les matières autres que le contentieux pénal, le Parquet près la Cour constitutionnelle intervient par voie d'avis motivé ou des observations. L'avis est une note écrite, présentant le point de vue soutenu du Parquet sur toute question de droit soulevée par une partie ou les parties au contentieux devant la Cour constitutionnelle. Tandis que les observations sont formulées de manière verbale à l'audience.

Certes, comme tous les avis du Ministère public, celui du Parquet général près la Cour constitutionnelle ne lie pas les juges constitutionnels dans la mesure où ceux-ci ne sont pas obligés de suivre le point de vue du Parquet. Dépourvus de caractère contraignant, les avis sont néanmoins obligatoires. Le défaut d'un avis ou lorsqu'il est donné dans des formes autres que celles prévues par la loi entraine l'irrégularité de la décision.

Il convient de signaler néanmoins qu'en matière de contentieux d'inconstitutionnalité, le Procureur Général près la Cour constitutionnelle a le pouvoir de saisir d'office celle-ci en inconstitutionnalité d'un acte législatif ou réglementaire lorsqu'il estime que ceux-ci portent atteinte aux droits fondamentaux de la personne humaine ou aux libertés publiques ${ }^{84}$.

\section{c. Rôle du Parquet dans l'exécution des arrêts ${ }^{85}$}

Les arrêts de la Cour constitutionnelle sont obligatoires et s'imposent aux pouvoirs publics, à toutes les autorités administratives, juridictionnelles, civiles, militaires ainsi qu'à tous les particuliers $^{86}$. Ils sont immédiatement exécutoires ${ }^{87}$. Le Procureur Général en poursuit l'exécution ${ }^{88}$. La loi organique n'indique pas clairement les actes que doit poser le Parquet

83 Article 14 de la Loi organique $n^{\circ} 13 / 026$, note 61.

84 Article 49 de la Loi organique $\mathrm{n}^{\circ} 13 / 026$ note 61.

85 Lire sur cette question, Mukiramfi Samba, «Réflexions prospectives sur un contentieux d'exécution des arrêts de la Cour constitutionnelle » Annuaire Congolais de Justice Constitutionnelle, Volume 2, 2017, p.28.

86 Article 95 alinéa 2 de la Loi organique, note 61.

87 Article 168 alinéa 2 de la Constitution, note 4.

88 Article 94 de la Loi organique $n^{\circ} 13 / 026$, note 61 . 
Général pour assurer l'exécution des arrêts de la Cour constitutionnelle. Le règlement intérieur du Parquet Général près la Cour constitutionnelle ne donne pas non plus de détails sur la mission exacte du Parquet en cette matière ${ }^{89}$.

Néanmoins, le code de procédure pénale est un peu plus explicite sur la question de l'exécution des jugements. En effet, l'exécution est poursuivie par le ministère public en ce qui concerne la peine de mort, la peine de servitude pénale, les dommages-intérêts prononcés d'office et la contrainte par corps; par la partie civile, en ce qui concerne les condamnations prononcées à sa requête; par le greffier, en ce qui concerne le recouvrement des amendes, des frais et du droit proportionnel ${ }^{90}$.

Si le jugement ne prononce pas l'arrestation immédiate, le Ministère public avertit le condamné à la servitude pénale qu'il aura à se mettre à sa disposition dans la huitaine qui suivra la condamnation devenue irrévocable. À l'expiration du délai imparti au condamné, le ministère public le fait appréhender au corps ${ }^{91}$.

Même dans le cas où l'arrestation immédiate n'a pas été ordonnée par le juge, le ministère public peut, à tout moment après le prononcé du jugement, faire arrêter le condamné si, à raison de circonstances graves et exceptionnelles, cette mesure est réclamée par la sécurité publique ou s'il existe des présomptions sérieuses que le condamné cherche et qu'il peut parvenir à se soustraire à l'exécution du jugement ${ }^{92}$. Le ministère public fait remettre le condamné au gardien de l'établissement où la peine doit être purgée; celui-ci délivre une attestation de la remise ${ }^{93}$.

Si le Parquet Général près la Cour constitutionnelle peut s'appuyer sur le droit commun de procédure pénale pour exécuter les arrêts de la Cour constitutionnelle en matière pénale, il n'existe pas des dispositions spécifiques sur l'exécution des arrêts de la Cour intervenus dans d'autres matières, notamment le contentieux d'inconstitutionnalité. Dans tous les cas, l'administration qui n'exécute pas les arrêts de Cour constitutionnelle s'expose à engager sa responsabilité.

\section{Conclusion}

Le Parquet est une institution omniprésente et incontournable dans l'administration de la justice en RDC. Sa structure éparpillée près chaque juridiction auprès de laquelle il joue le rôle du Ministère public ou d'organe de la loi, donne l'image d'une institution non organisée et non hiérarchisée. Depuis la suppression du parquet sur la liste des détenteurs du pou-

89 Règlement Intérieur du Parquet Général près la Cour constitutionnelle, J.O.R.D.C., Numéro spécial, juin 2018, pp.161-181.

90 Article 109 du Décret du 06 août 1959, portant Code de Procédure pénale tel que modifié et complété par la Loi nº6/019 du 20 juillet 2006, J0RDC, 47ème année, $n^{\circ} 15$ du $1^{\text {er }}$ aout 2006.

91 Article 110 du Décret du 06 août 1959, note 88;

92 Article 111 du Décret du 06 août 1959, note 88.

93 Article 112 du Décret du 06 août 1959, note 88. 
voir judiciaire suite à la révision constitutionnelle de 2011, cette institution est désormais créée par le législateur.

Le statut flottant du Parquet entre institution du pouvoir judiciaire et instrument du Gouvernement, rend les magistrats très vulnérables aux influences politiques. La mission du Parquet diffère selon que la juridiction a des compétences pénales ou pas. Son pouvoir de donner des avis sur chaque question de droit contribue ainsi à la bonne application de la loi.

Mais, les avis du Parquet pouvaient bien contribuer aussi au développent du droit s'ils étaient publiés in extenso avec la décision du juge pour être confrontée à celle-ci. De la sorte, les avis du Ministère public, souvent repris sur dispositif dans les jugements et arrêts et donc, moins connus du public et du monde scientifique deviendraient accessibles à tous.

\section{Bibliographie}

\section{A. Textes juridiques}

Règlement Intérieur du Parquet Général près la Cour constitutionnelle, J.O.R.D.C., Numéro spécial, juin 2018, pp.161-181.

Décret du 06 août 1959 portant code de procédure pénale, B.O. 1959, p.194.

Loi n⿳002/2001 du 03 juillet 2001 portant création, organisation et fonctionnement des Tribunaux de commerce, J.O.R.D.C., $42^{\mathrm{ème}}$ année, $\mathrm{n}^{\circ} 14$ du 15 juillet 2001, p.4.

Loi n ${ }^{\circ}$ 16-2002 du 16 octobre 2002 portant création, organisation et fonctionnement des Tribunaux du travail, J.O.R.D.C., $43^{\text {ème }}$ année, Numéro spécial.

Loi n ${ }^{\circ} 023-2002$ du 18 novembre 2002 portant code justice militaire, J.O.R.D.C., $43^{\text {ème }}$ année, Numéro spécial, du 20 mars 2003, p. 1.

Constitution de la RDC du 18 février 2006, J.O.RDC, 47ème année, Numéro spécial du 18 février 2006.

Loi organique $n^{\circ} 06 / 20$ du 10 octobre 2006 portant statut des magistrats telle modifiée par la Loi organique $\mathrm{n}^{\circ} 15 / 014$ du 1er août 2015, J.O.R.D.C., 56ème année, n 17, 1er septembre 2015.

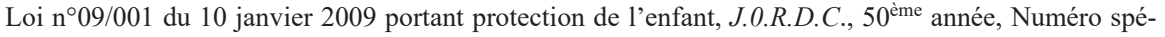
cial 12 janvier 2009, pp.13-48.

Loi n ${ }^{\circ}$ 11/002 du 20 janvier 2011 portant révision de certains articles de la Constitution de la Répu-

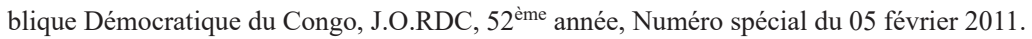

Loi organique $\mathrm{n}^{\circ}$ 13/011-B du 11 avril 2013 portant organisation, fonctionnement et compétences des juridictions de l'ordre judicaire, J.O.R.D.C., $54^{\text {ème }}$ année, Numéro spécial 7, juin 2018, pp.249-282.

Loi organique ${ }^{\circ} 13 / 026$ du 15 octobre 2013 portant organisation et fonctionnement de la Cour constitutionnelle, J.O.R.D.C., $59^{\text {ème }}$ année, Numéro spécial, juin 2018, pp.133-153.

Loi organique $\mathrm{n}^{\circ} 16 / 027$ du 15 octobre 2016 portant organisation, compétence et fonctionnement des juridictions de l'ordre administratif, J.O.R.D.C., $59^{\text {ème }}$ année, Numéro spécial du 7 juin 2018, pp.289-282. 


\section{B. Doctrine}

Antoine Rubbens, Droit judicaire congolais, Tome I, Le pouvoir, l'organisation et la compétence judicaires, Kinshasa/Bruxelles, 1970.

Auguste Mampuya Kanunk'a Tshiabo, « Au feu! L'article 220 est violé! », Le Phare du 13 janvier 2011 (Quotidien paraissant à Kinshasa).

Bayona-ba-Meya, "L'indépendance de la magistrature en République du Zaïre », Annales de la Faculté de droit, Vol. I, 1972.

Emmanuel J. Luzolo Bambi Lessa, Traité de droit judiciaire. La justice congolaise et ses institutions, Kinshasa, 2018.

Emmanuel -Janvier Luzolu Bambi Lessa et Nicolas Abel Bayona Ba Meya, Manuel de Procédure pénale, Kinshasa, 2011.

Guy-Prosper Djuma Bilali Lokema, «Parquet Général près la Cour constitutionnelle de la République Démocratique du Congo », Annuaire Congolais de Justice Constitutionnelle, Vol.1, 2016, p.p.89-110.

Jean-Louis Essambo Kangashe, La Constitution congolaise du 18 février 2006 à l'épreuve du constitutionnalisme, contraintes pratiques et perspectives, 2010.

Marcel Wetsh'okonda Koso, " Nature juridique et garanties d'indépendance des officiers du ministère public sous l'empire de la Constitution congolaise du 18 février 2006 telle que révisée par la loi de révision constitutionnelle du 20 janvier 2011 », Librairie Africaine d'Etudes Juridiques, 2017.

Marcel Wetsh'okonda Koso, La protection des droits de l'homme par le juge constitutionnel congolais. Analyse critique et jurisprudence (2003-2013), Paris, 2016.

Mukiramfi Samba, "Réflexions prospectives sur un contentieux d'exécution des arrêts de la Cour constitutionnelle » Annuaire Congolais de Justice Constitutionnelle, Volume 2, 2017, pp.27-56.

Nkongolo Tshilengu, Droit judicaire congolais. Le rôle des cours et tribunaux dans la restauration d'un droit violé ou contesté, Kinshasa, 2003. 